\title{
Experimental study of intermediate length coupling beams subjected to monotonic load
}

\begin{abstract}
Experimental study is conducted on four reinforced concrete coupling beams, with length to depth ratios of 2.5 and 3.1. The beams are designed to fail in shear after yielding of longitudinal reinforcement. The longitudinal reinforcement contents are 0.76 and 1.14 percent, while shear reinforcement varies from 0.2 to 0.5 percent. The beams are loaded monotonically until failure. It is found that the shear stiffness of such beams reduces to 0.1 of the initial stiffness upon yielding of reinforcement. An empirical equation is proposed to assess the shear strength degradation of the beams based on axial strain of longitudinal reinforcement.
\end{abstract}

Keyword: Experimental investigation; Reinforced Concrete (RC) structure; Quasi-static 